\title{
Design of DPX system for real-time spectrum analysis and signal detection
}

\author{
Shijian Guo ${ }^{\text {a) }}$, Jian Su, Gang Sun, Zhiyong Zhao, \\ and Zengping Chen \\ ATR Key Lab, National University of Defense Technology, \\ Changsha, Hunan, 410073, P. R. China \\ a)sjguo@nudt.edu.cn
}

Abstract: A new method named Digital Phosphor technology (DPX) is presented for real-time spectrum analysis and signal detection. The process of generating the 3-D spectrum in the DPX system is analyzed, and a practical DPX system is designed and implemented. The maximum effective bandwidth is $500 \mathrm{MHz}$, and the signals are sampled directly in radio frequency $(\mathrm{RF})$ with $1.2 \mathrm{GHz}$ sampling rate. The main data processing module employs dual-FPGA architecture. 65,535 frames of frequency waveforms are continuously processed and accumulated in one DPX image within $100 \mathrm{~ms}$. The experimental results have indicated that the 3-D spectrum of DPX greatly improves the capabilities of signal discovery, capture and analysis, and the DPX system is adapted to real-time spectrum analysis and signal detection.

Keywords: Digital Phosphor technology (DPX), system design, real-time spectrum analysis, signal detection

Classification: Electron devices, circuits, and systems

\section{References}

[1] Tektronix: Fundamentals of Real-Time Spectrum Analysis (2009).

[2] Tektronix: DPX Acquisition Technology for Spectrum Analyzers Fundamentals Primer (2009).

[3] Rohde and Schwarz: Implementation of Real-Time Spectrum Analysis (2011).

[4] J. D. Schwartz, J. Azana and D. V. Plant: IEEE Microw. Wireless Compon. Lett. 16 [4] (2006) 215.

[5] V. Teppati, A. Ferrero and M. Sayed: Modern RF and Microwave Measurement Techniques (Cambridge University Press, 2013).

[6] Z. P. Chen, J. H. Wu, S. Y. Su and S. J. Guo: Signal Processing 28 [2] (2012).

[7] U. Meyer-Baese: Digital signal processing with field programmable gate arrays (Springer Verlag, 2004) 2nd ed.

[8] C. J. Kief, M. S. Pattichis, L. H. Pollard, G. A. Vera and J. E. Parra: Comput. Appl. Eng. Educ. 17 [2] (2009) 232.

[9] E. Çetin, R. C. S. Morling and I. Kale: IEEE Proc. Instrumentation and Measurement Technology Conference 1 (1997) 96.

[10] J. Palmer and B. Nelson: Lecture Notes Comput. Sci. 3203 (2004) 948.

[11] M. Ayinala, M. Brown and K. K. Parhi: IEEE Trans. Very Large Scale 
Integr. (VLSI) Syst. 20 [6] (2012) 1068.

[12] J. García, J. A. Michell, G. Ruiz and A. M. Burón: Proc. SPIE 6590 (2007) 1.

\section{Introduction}

Digital Phosphor technology (DPX) is an innovative technology brought forward firstly by the Tektronix Company [1,2], and is developed fast and widely used in real-time spectrum analysis and signal detection. The DPX 3 -d spectrum is a spectrum image, not a line, and displays multiple signals appeared at continuous time in a same image. The image is updating continuously and the display time of an image is settled and longer relative to the existing time of transient signal, corresponding to prolong the display time of transient signal, so the transient signal can be captured. The DPX image that one see on screen is composed of pixels representing $\mathrm{x}$, $\mathrm{y}$, and $\mathrm{z}$ values for frequency, amplitude, and "hit count" (some instruments adopt "density" in place of "hit count"), and use different color to represent the different occurrence counts of the signal $[1,2,3]$, so the rare transients can visually be distinguished from normal signals and background noise. Fig. 1 shows the comparison between the DPX technology and traditional spectrum to analyze the WIFI signal transmitted by the mobile telephone at the same time. The RF signals transmit in periodic, transient, and parameters like frequency, power levels, and modulation formats are constantly in flux. All these factors combine to make it tough to see what's really going on in the traditional spectrum. The DPX image accumulates large numbers of spectrums of a period of time and provides 3-D, colorful and intuitive signal display. And with DPX, the signals that can't see in the traditional spectrum can be discovered. The DPX image displays an unprecedented amount of information. With a more detailed picture of signal, the DPX greatly improves the capabilities of signal discovery, trigger, capture and analysis. It is well suited for application in the realtime spectrum analysis and signal detection, and has found an increasingly wide utilization in many fields such as electronic reconnaissance and disturber diagnosis $[4,5,6]$. To apply the DPX, the process of generating the DPX images is analyzed and a practical DPX system is designed and implemented referring other electron systems [7, 8, 9, 10, 11, 12].

\section{Process Analysis of DPX 3-D Spectrum Generating and System Design}

\subsection{Process of generating the DPX 3-D spectrum}

Fig. 2-Fig. 4 show the process that generates the DPX images. The digitized data stream is chopped into data records, and then an FFT is performed on each record, continually producing spectral waveforms, as show in Fig. 2 (a). The spectral waveforms are plotted onto a grid of counting cells called the "bitmap database", shown in Fig. 2 (b). As a dense grid, the bitmap database is created by dividing a spectrum graph, and its columns represent the trace of amplitude values and rows for points on the frequency axis. A waveform contains one amplitude value for each 


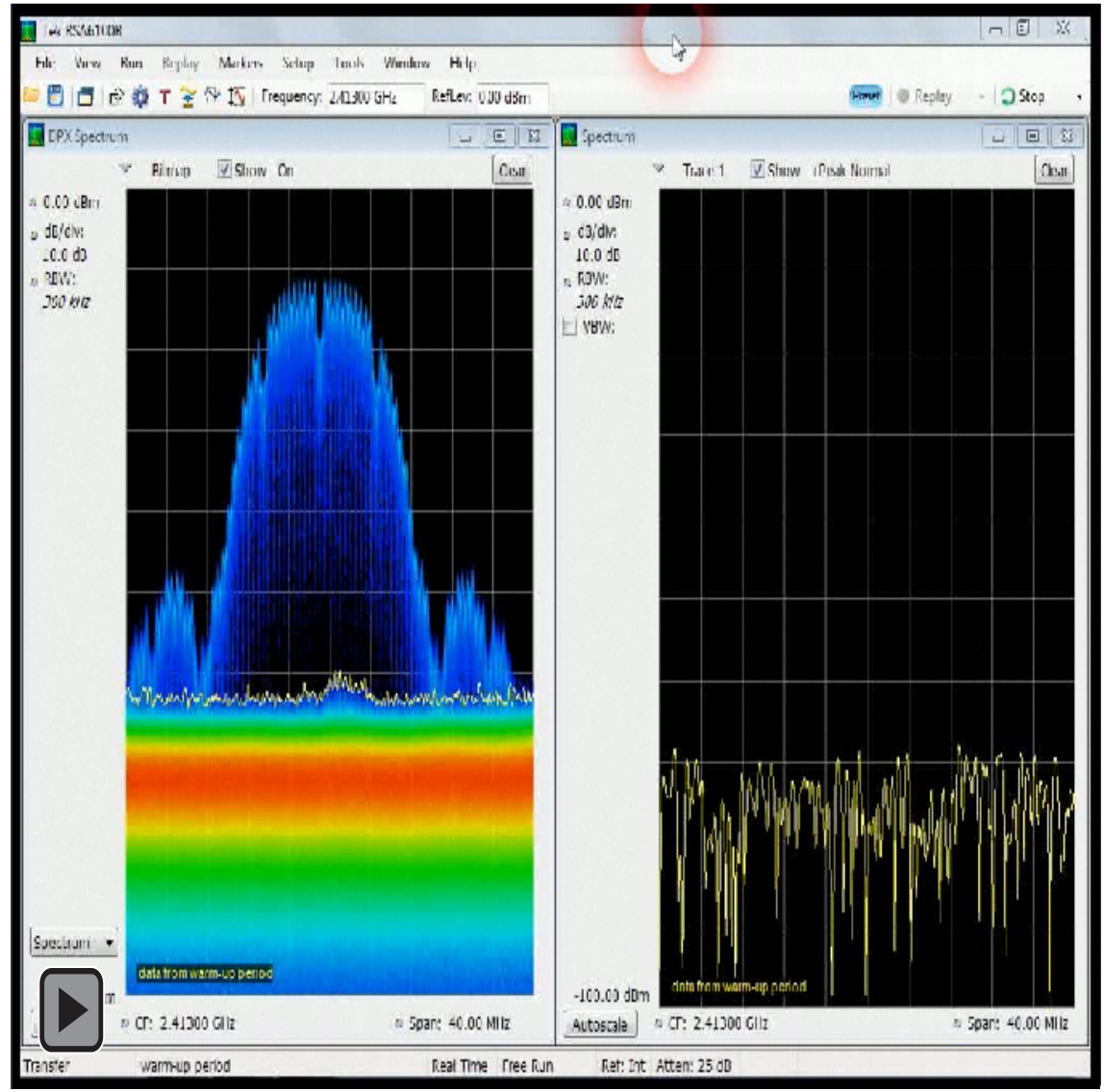

Fig. 1. Comparison between the DPX technology and traditional spectrum to analyze the WIFI signal transmitted by the mobile telephone at the same time (movie)

frequency [1]. The actual 3-D database in a DPX system for real-time spectrum analyzer may contain many columns and rows, but an $11 \times 10$ matrix is used to illustrate the concept. So the spectral waveforms will each contain $11 \times 10$ points.

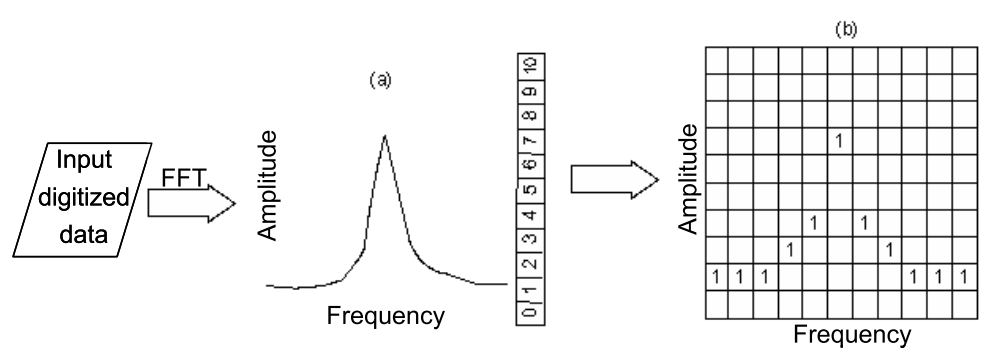

Fig. 2. Example of bitmap database (b) after FFT (a)

(c) IEICE 2013

DOI: $10.1587 /$ elex.10.20130657 Received August 20, 2013

Accepted September 04, 2013

Publicized September 13, 2013

Copyedited October 10, 2013 
them yet. Fig. 3 (b) shows values that the simplified database might contain after an additional eight spectral transforms have been performed and their results stored in the cells. One of the nine spectrums happened to be sampled at a time during which the signal was absent, as can be seen by the string of " 1 " values at the noise floor [2].

(a) 9 FFT Spectrums $\Rightarrow \sum \Longrightarrow$ (b) Pixel Occurrence Histogram
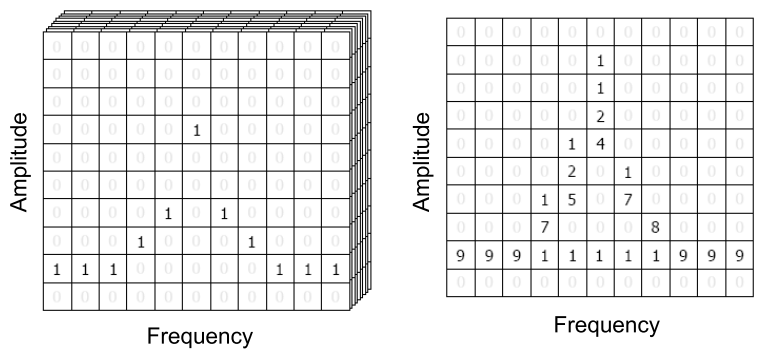

Frequency

Fig. 3. Example of 3-D bitmap database (a) and 9 accumulated spectrums updates (b). Note that each column contains the same total number of "hits"

When the number of occurrences value is mapped to a color scale, shown in Fig. 4 (a), the accumulated data turns into intuitive visual information. The table shows the color mapping algorithm that is used for this example. Warmer colors (red, orange, yellow) indicate more occurrences [2]. The picture of Fig. 4 (b) is the result of coloring the database cells according to how many times they were written into by the nine spectrums. Displaying these colorful cells, one pixel per cell on the screen, creates the spectacular DPX displays, example is shown in Fig. 4 (c). The color displays in the DPX images is proportional to the occurrence counts, so we can visually distinguish rare transients from normal signals and background noise.

(a) Color Map

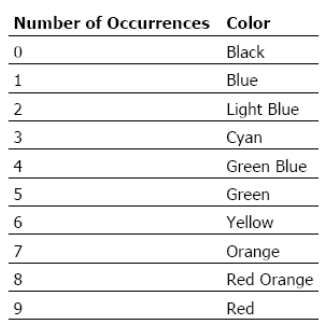

(b) DPX Color Display

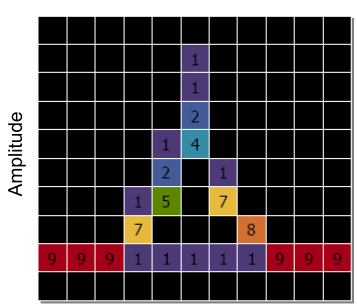

Frequency (c) Real DPX 3-D spectrum image

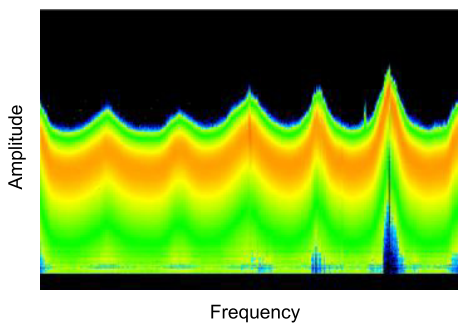

Fig. 4. Example Color mapping algorithm (a), Color coded low-resolution example (b), and a real DPX display (c)

\subsection{System design}

Fig. 5 shows the block diagram of DPX system. Excluding the antenna, the hardware consists of the radio frequency processing module (RFPM) and the data processing module (DPM). The two hardware modules receive the signal, calculate the frequency waveform and accumulate the spectrum for DPX. The host is an industrial control computer (ICC), receives and displays the DPX 3-D spectrum, and sends out the control commands 


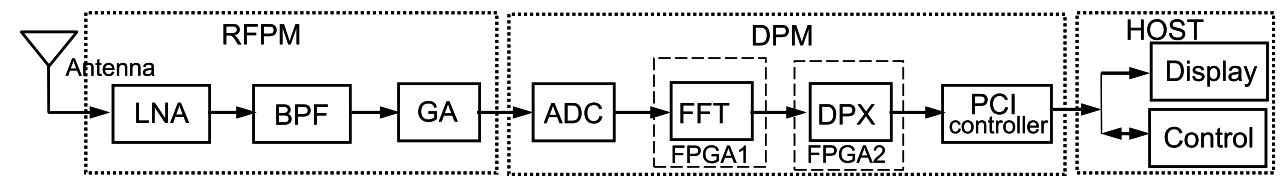

Fig. 5. DPX system block diagram

though the CPCI interface.

To cut the cost and predigest the structure, the analog signals are direct sampling in RF. The RFPM is implemented only with a low-noise amplifier (LNA), a bandpass filter (BPF) and a gain adjustment (GA). The RF signal is adjusted to the appropriate amplitude range and delivered to DPM. Fig. 6 shows the physical photograph of RFPM.

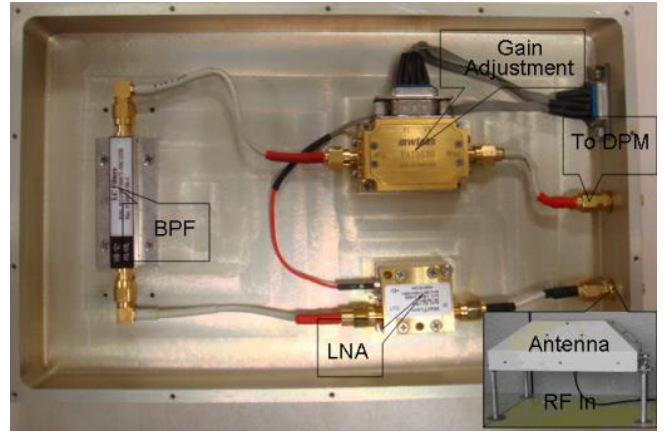

Fig. 6. Physical photograph of RFPM

Fig. 7 shows the DPM block diagram. The main data processing unit employs dual-FPGA architecture [8]. The input analog signal is digitized by the A/D converter (AT84AS003, $3 \mathrm{GHz}$ analog input bandwidth, $1.5 \mathrm{GHz}$ sampling rate maximum, 10-bit), the digital signal is sent to FPGA1 (XC5VSX95T, 14,720 slices, 8,784 kb BRAM, 640 IO) to calculate the frequency waveform applying the FFT arithmetic [9, 10, 11, 12], and the result is transmitted to FPGA2 (XC4VFX100, 42,176 slices, $6,768 \mathrm{~kb}$ BRAM, 2 embedded PowerPC processor cores, 768 IO) for the accumulation of the 3-D spectrum. The two FPGAs are interconnected through a self-defined bus. The FPGA2 exchanges data with the mainboard through the CPCI bus using an external PCI controller.

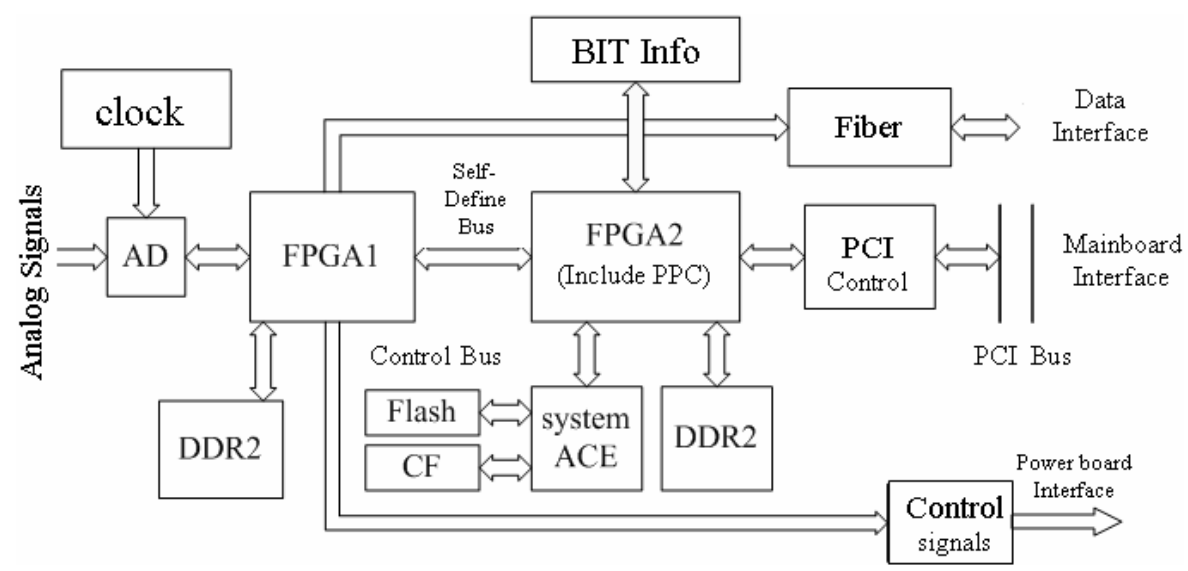

Fig. 7. DPM block diagram 
Fig. 8 shows the physical photograph of DPM board. The FPGA1 includes an external memory slot, and can be accessed a 2GByte DDR2 memory as the data cache. The FPGA1 also includes an external 4-access fiber interface to exchange data with other devices. The FPGA2 is also connected with a 2GByte DDR2 memory, and at the same time a large capacity CF card and a FLASH are connected to FPGA2 via System ACE controller $[7,8,12]$. The high-speed sampling clock is generated by the board. The board information test (BIT) is collected by the FPGA2, and then transmitted to the host.

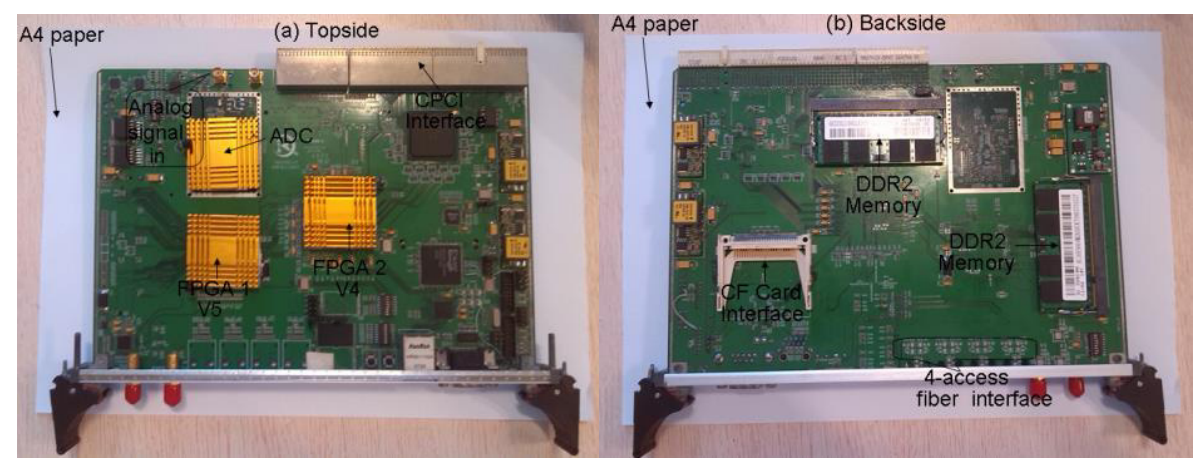

Fig. 8. Physical photograph of DPM board. (a) is the topside, (b) is the backside

\subsection{System characteristic}

The signals are sampled directly in RF with $1.2 \mathrm{GHz}$ sampling rate, and the maximum effective bandwidth is $500 \mathrm{MHz}$. 65,535 frames of frequency waveforms are continuously processed and accumulated in one DPX image within $100 \mathrm{~ms}$. The main performance parameters comparing with the Tektronix Company's spectrum analyzer of RSA6000 [1,2] are shown in Table I. The system we designed has wider bandwidth, faster data processing rate, larger save capacity, and better time distinguish, but the frequency distinguish is worse because of the so wide bandwidth.

Table I. Performance parameters of DPX system (Tektronix Company's spectrum analyzer RSA6000 in the bracket)

\begin{tabular}{c|c|c|c|c|c}
\hline Sampling rate & $\begin{array}{c}\text { Effective } \\
\text { bandwidth }\end{array}$ & $\begin{array}{c}\text { Max spectrum } \\
\text { accumulate rate }\end{array}$ & $\begin{array}{c}\text { Save } \\
\text { capacity }\end{array}$ & $\begin{array}{c}\text { Least time } \\
\text { distinguish }\end{array}$ & $\begin{array}{c}\text { Frequency } \\
\text { distinguish }\end{array}$ \\
\hline $1.2 \mathrm{GHz}$ & $500 \mathrm{MHz}$ & $655360 / \mathrm{s}$ & $\begin{array}{c}\mathrm{GB} \\
(1 \mathrm{~GB})\end{array}$ & $\begin{array}{c}5 \mathrm{~ns} \\
(20 \mathrm{~ns})\end{array}$ & $\begin{array}{c}40 \mathrm{kHz} \\
(10 \mathrm{kHz})\end{array}$ \\
\hline
\end{tabular}

Not only is a spectrum analyzer, the system designed but also mainly for the signal automatic detection. Comparing with other signal detection system, the applying of DPX technology in our system achieves more characteristics, and some examples are shown in the experiments. Based on the DPX, other technologies are also applied for signal detection just like FMM (Frequency Mask Matching), DT (Density Trigger), DIP (Digital Image Processing) and PR (Pattern Recognition) [6] combined with the traditional time domain, frequency domain and time-frequency domain signal detection technology. 
The effective bandwidth is $500 \mathrm{MHz}$, and the high-speed sample rate of 1.2 $\mathrm{GHz}$ brings massive account of data, at the same time, the arithmetic for signal detection is excessive and complex, therefore, dual-FPGA architecture is applied to achieve greater processing performances. The work is divided to the two FPGAs in proportion, and the program is optimized to satisfy the need of real-time.

To make a summary, comparing with other systems, our system has significant improvements:

- New arithmetic based on the DPX for signal detection

- Less RF components because of direct sampling in RF

- Wideband real-time spectrum analysis performance of $500 \mathrm{MHz}$

- Dual-FPGA architecture to achieve real-time massive account of data processing

\section{Experimental results}

\subsection{Disturber analysis}

Fig. 9 (a) shows the 1-D range profile of a certain wideband imaging radar received signal. Exclude the echo signal, there are also two disturbers consisting in the figure, shown in the black pane. To check out the sources of disturber, the DPX equipment is used to observe the spectrum. Fig. 9 (b) shows the DPX 3-D spectrum of the received signal. Two disturbers are dug out, shown in the black pane. The left pane is a signal of fixed frequency, and the right pane is a wideband disturbed signal or distortion. The two disturbed signals are covered by the wideband radar echo signal. To ascertain the two disturbers further, the time-frequency distribution analysis result is also integrated, shown in Fig. 9 (c). The left pane shows the fixed disturbed signal that persists about $50 \mu \mathrm{s}$, and the right pane shows the wideband disturbed signals which are two LMF signals with different modulated frequency slope.
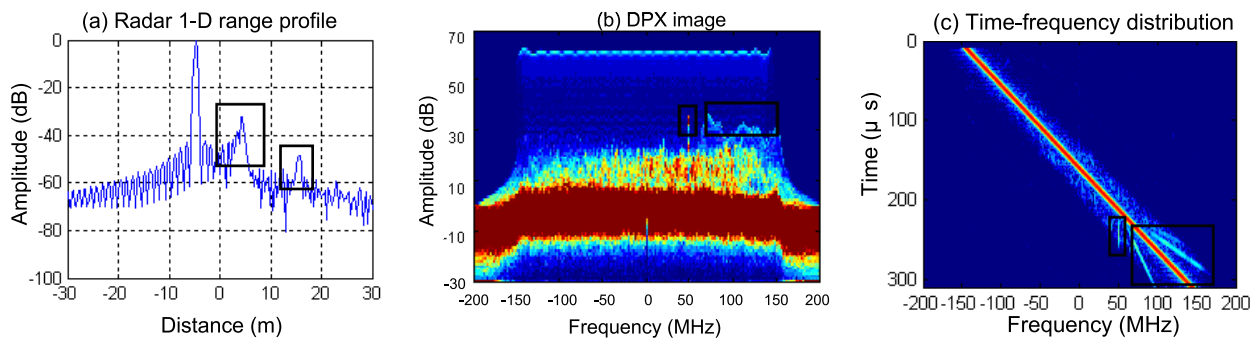

Fig. 9. Distortion of 1-D range profile of a certain wideband imaging radar (a), DPX 3-D spectrum

(b) and time-frequency distribution image (c)

\subsection{Signal detection}

A received signal $s(t)=s_{1}(t)+s_{2}(t), s_{1}(t)$ and $s_{2}(t)$ are both pulse LMF signals, and the expression as follow:

$$
\left\{\begin{array}{l}
s_{1}(t)=A_{1} \exp \left(j \pi B_{1} t^{2} / \tau_{1}\right) \\
s_{2}(t)=A_{2} \exp \left(j \pi B_{2} t^{2} / \tau_{2}\right)
\end{array} .\right.
$$


are shown in Table II. Fig. 10 (a) and (b) shows the waveforms of the time domain and frequency domain of $s(t)$. The $s_{2}(t)$ is "sheltered" from $s_{1}(t)$ and can not be discovered.

Table II. Parameters of $s_{1}(t)$ and $s_{2}(t)$

\begin{tabular}{c|c|c|c|c}
\hline Signal & Amplitude & Time width & Bandwidth & Repetition time \\
\hline$s_{1}(t)$ & $A_{1}=200$ & $\tau_{1}=75 \mu \mathrm{s}$ & $B_{1}=300 \mathrm{MHz}$ & $1 \mathrm{~ms}$ \\
\hline$s_{2}(t)$ & $A_{2}=2$ & $\tau_{2}=75 \mu \mathrm{s}$ & $B_{2}=10 \mathrm{MHz}$ & $10 \mathrm{~ms}$ \\
\hline
\end{tabular}

(a) Time domain
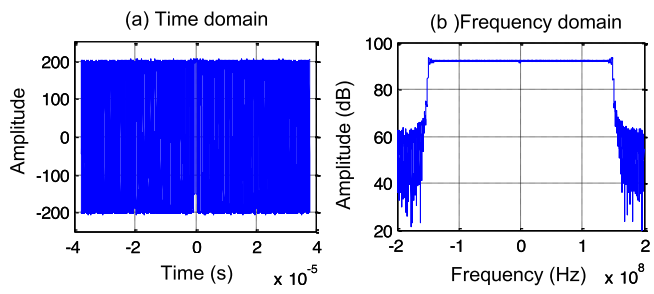

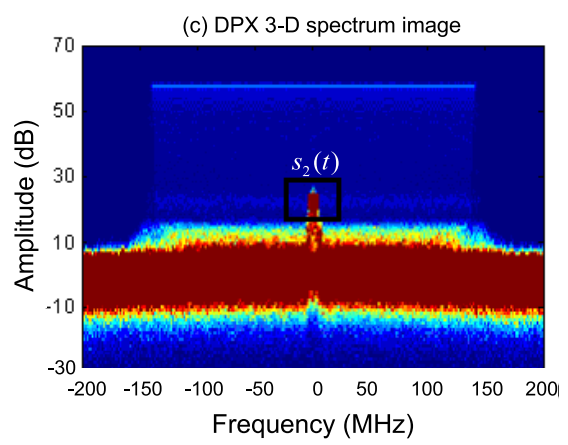

Fig. 10. Simulation of the time domain (a) and frequency domain (b) of $s(t)$, and DPX 3-D spectrum image for $s_{1}(t)$ and $s_{2}(t)$ that spectrum mixed $(\mathrm{c})$.

Fig. 10 (c) shows the DPX 3-D spectrum image. The color that represents the hit count is different because the signal's repetition time is different, so the weak signal $s_{2}(t)$ hidden in signal $s_{1}(t)$ can be detected, shown in the black pane. This has shown the advantage of the DPX in signal detection.

\section{Conclusions}

DPX is well suited for the real-time spectrum analysis and signal detection. The process of generating the 3-D spectrum in the DPX system is analyzed, and a practical DPX system is implemented. The reconnaissance bandwidth is $500 \mathrm{MHz}$, the sampling rate of $1.2 \mathrm{GHz}$ brings massive account of data, and the arithmetic for signal detection is excessive and complex, therefore, dual-FPGA architecture is applied to achieve greater processing performances. The work is divided to the two FPGAs in proportion, and the program is optimized to satisfy the need of real-time. The experimental results have indicated that the DPX system that we have implemented is feasible in practice, and improves the capabilities of signal discovery, analysis and detection. 